\title{
Diffuse large B-cell lymphoma with distinctive patterns of splenic and bone marrow involvement: clinicopathologic features of two cases
}

\author{
William G Morice, Fausto J Rodriguez, James D Hoyer and Paul J Kurtin \\ Department of Laboratory Medicine and Pathology, Mayo Clinic, Rochester, MN, USA
}

\begin{abstract}
Two unusual cases of large B-cell lymphoma with predominant splenic and bone marrow (BM) involvement and similar clinical and histopathologic features are described. Both patients presented with nonspecific constitutional symptoms, unexplained cytopenias, and splenomegaly. Splenectomy revealed diffuse red pulp involvement by large B-cell lymphoma. The perisplenic lymph nodes were also involved diffusely with effacement of normal nodal architecture, excluding a diagnosis of intravascular large B-cell lymphoma. BM biopsies revealed striking erythroid hyperplasia without overt morphologic evidence of involvement by lymphoma. Immunoperoxidase staining of the marrow biopsies with antibodies to CD20 and erythroidassociated antigens revealed involvement by large B-cell lymphoma morphologically resembling the early pronormoblasts. In both cases there was prominent, but not exclusive, intravascular/intrasinusoidal lymphomatous marrow infiltration. These cases represent an unusual variant of large B-cell lymphoma with distinctive patterns of splenic and BM involvement. Furthermore, they underscore the difficulties in identifying intrasinusoidal marrow infiltration by lymphoma in H\&E-stained biopsy slides and demonstrate that this pattern of marrow infiltration may be seen in cases of large B-cell lymphoma distinct from the intravascular variant. Modern Pathology (2005) 18, 495-502, advance online publication, 15 October 2004; doi:10.1038/modpathol.3800297
\end{abstract}

Keywords: diffuse large B-cell lymphoma; intravascular lymphoma; spleen; bone marrow; erythroid hyperplasia; non-Hodgkin lymphoma

Diffuse large B-cell lymphoma (LBCL) can involve nodal or extranodal sites; however, initial presentation in the spleen occurs infrequently. ${ }^{1}$ Typically when diffuse LBCL involves the spleen it forms discrete tumoral masses within the splenic parenchyma. ${ }^{2}$ A recent report detailed other less common patterns of splenic involvement by LBCL including diffuse infiltration of the splenic red pulp. ${ }^{3}$ The clinicopathologic features of diffuse LBCL with these unusual patterns of splenic involvement have yet to be fully elucidated. Described herein are the distinctive clinical and histopathologic features of two cases of splenic LBCL with diffuse red pulp infiltration and concurrent involvement of lymph nodes and bone marrow (BM). These cases had

Correspondence: Dr WG Morice, MD, PhD, Department of Laboratory Medicine and Pathology, Mayo Clinic, Rochester, MN, USA.

E-mail: morice.william@mayo.edu

Received 8 June 2004; revised 20 August 2004; accepted 23 August 2004; published online 15 October 2004 strikingly similar clinical presentations chiefly characterized by dyspnea, unexplained cytopenias, and splenomegaly. In both instances, splenectomy performed to alleviate the cytopenias revealed diffuse, homogeneous involvement of the splenic red pulp by diffuse LBCL. Although the pattern of splenic red pulp involvement and the clinical features raised the possibility of intravascular LBCL, in both cases there was parenchymal involvement of splenic hilar lymph nodes, excluding this diagnosis as defined by the most recent WHO criteria. ${ }^{4}$ Furthermore, both cases had prominent BM involvement by lymphoma, which was difficult to detect by routine morphologic evaluation of hematoxylin and eosin (H\&E)-stained biopsy slides. This difficulty was due both to the presence of prominent erythroid hyperplasia as well as a predominant, but not exclusive, intrasinusoidal pattern of marrow infiltration. These cases appear to be representative of an uncommon variant of diffuse LBCL, which is difficult to recognize by clinical, radiologic and BM pathologic features. In addition, these cases 
illustrate that intrasinusoidal marrow infiltration by LBCL may be seen in disorders distinct from intravascular variant.

\section{Materials and methods}

Clinical information was abstracted from medical chart review. Peripheral blood smear and BM aspirates were stained by a modified Giemsa method. Representative sections of the spleen and lymph node specimens were fixed in formalin or B5; the BM biopsies were fixed in B5 and subsequently decalcified by a nitric acid-based method. Immunoperoxidase stains were performed on the paraffin sections of the spleen, lymph node, and marrow tissues according to previously described techniques. $^{5}$ The spleen and BM biopsies were stained with antibodies to CD3 (Cell Marque, dilution 1:300), CD20 (Dako, 1:60), CD34 (Becton Dickinson, 1:50), and Factor VIII (Dako, 1:1000); splenic sections were also stained with antibodies to CD5 (Novocastra, 1:20), CD10 (Novocastra, 1:10), CD138 (Dako, 1:50), BCL-2 (Dako, 1:50), BCL-6 (Dako 1:50), cyclin D1 (Dako 1:800, patient 2 only), tartrate resistant acid phosphatase (TRAP, Zymed Kit), DBA-44 (Dako 1:100), MUM-1 (Dako 1:50), and human herpes virus 8 (HHV-8, Novocastra 1:200). Additional immunoperoxidase stains were performed on paraffin sections of the BM biopsies using antibodies to glycophorin 1A (Dako, 1:100) hemoglobin A (Dako, 1:800). Paraffin sections of the lymph node biopsies were stained with antibodies to CD3 and CD20.

Flow cytometric immunophenotyping studies were performed in accordance with previously described methods on fresh splenic tissue and BM aspirate specimens using fluorochrome-conjugated antibodies against the following antigens: CD3, CD5, CD10, CD11c, CD14, CD16, CD19, CD20, CD22, CD23, CD45, kappa and lambda immunoglobulin light chains. ${ }^{6}$ The data were acquired on a FACSCaliber instrument and analyzed using Cell Quest software.
In situ hybridization was performed on paraffin sections of the splenectomy specimens using probes specific for Epstein-Barr virus-encoded RNA (EBER) according to previously described methods. ${ }^{7}$

This study was approved by the Mayo Foundation IRB and the patients consented to the review of their clinical histories and use of their materials.

\section{Results}

\section{Clinical and Laboratory Features}

The clinical, laboratory, and gross pathologic features are summarized in Table 1. Both patients presented with dyspnea and nonspecific constitutional symptoms including fatigue, weight loss, and night sweats. Marked splenomegaly, as noted both by physical examination and CT imaging (Figure 1), was the most remarkable finding in each case. Lymphadenopathy was not a prominent feature; moderate enlargement of mediastinal lymph nodes was detected by radiologic imaging alone in patient 1 . In both cases the serum LDH was elevated and CBC analysis revealed normocytic anemia with reticulocytosis and thrombocytopenia. Antiglycoprotein Ia/IIa antibodies were identified in the serum of patient 1; however, a platelet-bound antibody assay was negative and there was no other serologic evidence of antibodymediated red blood cell or platelet destruction in either case.

\section{Spleen and Lymph Node Pathology}

Splenectomy confirmed the presence of splenomegaly in both cases, with splenic weights of 1260 and $2050 \mathrm{~g}$ in patients 1 and 2, respectively (Table 1). The splenic cut surfaces revealed diffuse red pulp expansion without discrete masses or increased prominence of the white pulp.

Histologic examination of H\&E-stained splenic sections revealed diffuse infiltration of the red

Table 1 Clinicopathologic features

Patient 1

75

Age (years)

Gender

B-symptoms

LDH, U/l, serum (normal range 98-221 U/l)

$\mathrm{Hgb}(\mathrm{g} / \mathrm{dl})$

WBC $\left(\times 10^{9} / 1\right)$

Lymphocyte count $\left(\times 10^{9} / 1\right)$

Platelet count $\left(\times 10^{9} / 1\right)$

Splenomegaly (physical examination)

Spleen size (weight, measurements)

Lymphadenopathy (physical examination)

Nodal involvement (pathologic)
Male

Present

874

9.3

7.7

1.54

18

Present

$1260 \mathrm{~g}, 24 \times 15 \times 7 \mathrm{~cm}$

Absent

Splenic hilar
Patient 2

51
Male
Present
1711
10.5
5.3
0.79
33
Present
2050 g, $25 \times 20 \times 9 \mathrm{~cm}$
Absent
Splenic hilar

LDH, lactate dehydrogenase; Hgb, hemoglobin; WBC, white blood cell count. 


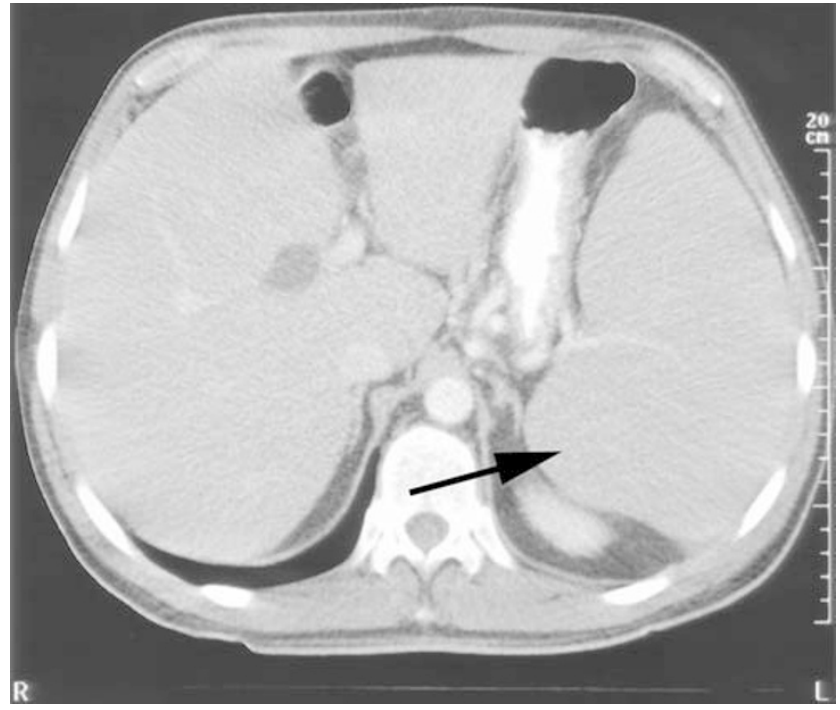

Figure 1 Radiographic imaging studies. Abdominal CT scan (from Patient 1) reveals marked splenic enlargement (arrow) with homogenous radiographic texture. No intrasplenic mass lesion or significant intra-abdominal adenopathy is detected.

pulp by neoplastic cells with large nuclei having vesicular chromatin, multiple nucleoli, and variably irregular nuclear contours (Figure 2a and b). These cells filled the red pulp cords and also infiltrated the sinusoids, many of which were still patent. Immunoperoxidase stains performed on paraffinembedded splenic tissue revealed the neoplastic cells to be CD20-positive B-cells, this immunostain also highlighted the infiltration of splenic cords and sinusoids (Figure 2c). The presence of malignant cells within vascular structures was also demonstrated by immunoperoxidase staining with antibodies to factor VIII (Figure 2d). In both cases, the neoplastic cells stained with antibodies to bcl-2 and MUM-1 but were negative for CD138, the follicular center cell-associated antigens CD10 and bcl-6, and HHV-8 (data not shown). This immunophenotype matches that previously ascribed to postgerminal center diffuse LBCL. ${ }^{8}$ The malignant cells were negative for the hairy cell leukemiaassociated antigens TRAP and DBA-44 by immunoperoxidase studies (data not shown). Flow cytometry performed on fresh splenic tissue demonstrated the presence of a kappa immunoglobulin light chain restricted, CD19 and CD20 positive B-cell population in both cases (data not shown). Neither case was CD10-positive by flow cytometry; in patient 1 a subset of the neoplastic cells showed weak coexpression of CD5 by this technique. Although CD5 expression was not detected by immunoperoxidase staining in either case, in light of the flow cytometry results, immunoperoxidase staining was performed on splenic tissue from patient 1 using antibodies to cyclin D1, the neoplastic cells were negative. Neither case showed evidence of EBV positivity by in situ hybridization using probes specific for EBV-encoded RNA (EBER) (data not shown).

Splenic hilar lymph nodes sampled at the time of splenectomy in both cases were involved by diffuse LBCL. In both instances, the neoplastic cells infiltrated the lymph node parenchyma with relative sparing of the sinuses (Figure 3a and b). This pattern of nodal involvement was confirmed by CD20 immunoperoxidase staining and essentially excluded a diagnosis of intravascular LBCL as defined by WHO criteria.

\section{Peripheral Blood and BM Pathology}

The peripheral blood smears of both patients showed changes compatible with peripheral loss and/or destruction of red blood cells and platelets including increased numbers of polychromatophilic red blood cells, a leukoerythroblastic picture, and moderate to marked thrombocytopenia with morphologically normal platelets. Occasional atypical lymphoid cells with intermediate to large-sized nuclei, irregular nuclear contours, and densely basophilic cytoplasm were also present. Peripheral blood involvement by lymphoma was confirmed by flow cytometric immunophenotyping in patient 1.

BM examination was performed prior to splenectomy in both cases to further evaluate the potential causes of the observed cytopenias. The BM findings are summarized in Table 2. In both cases the marrows were hypercellular and showed prominent, left-shifted, erythroid hyperplasia (Figure 4a and 4b). Large lymphoma cells were present in the marrow aspirates although they were exceedingly difficult to identify in the Wright-Giemsa-stained slides due to their resemblance to the early pronormoblasts. In both patients, the presence of lymphoma in the aspirates was confirmed by flow cytometry (data not shown).

The BM biopsies were involved by diffuse LBCL which, as in the aspirates, was difficult to identify by morphologic review of H\&E-stained slides (Figure 4a and b). The presence of diffuse LBCL in the marrow biopsies was obscured both by their resemblance to early pronormoblasts, which were increased in number, and the predominant but not exclusive intrasinusoidal pattern of lymphomatous marrow infiltration. The mixed intrasinusoidal and interstitial pattern of marrow involvement by diffuse LBCL was highlighted by immunoperoxidase staining with antibodies to CD20, which revealed both linear, intravascular staining as well as interstitial nodules of neoplastic cells (Figure 4c and d). The presence of atypical cells within vascular structures was also illustrated by immunostaining of endothelial cells (CD34 and Factor VIII, Figure 4e). Immunostaining of the biopsies with antibodies to the erythrocyte-associated antigens hemoglobin A and glycophorin $1 \mathrm{~A}$ confirmed the presence of 

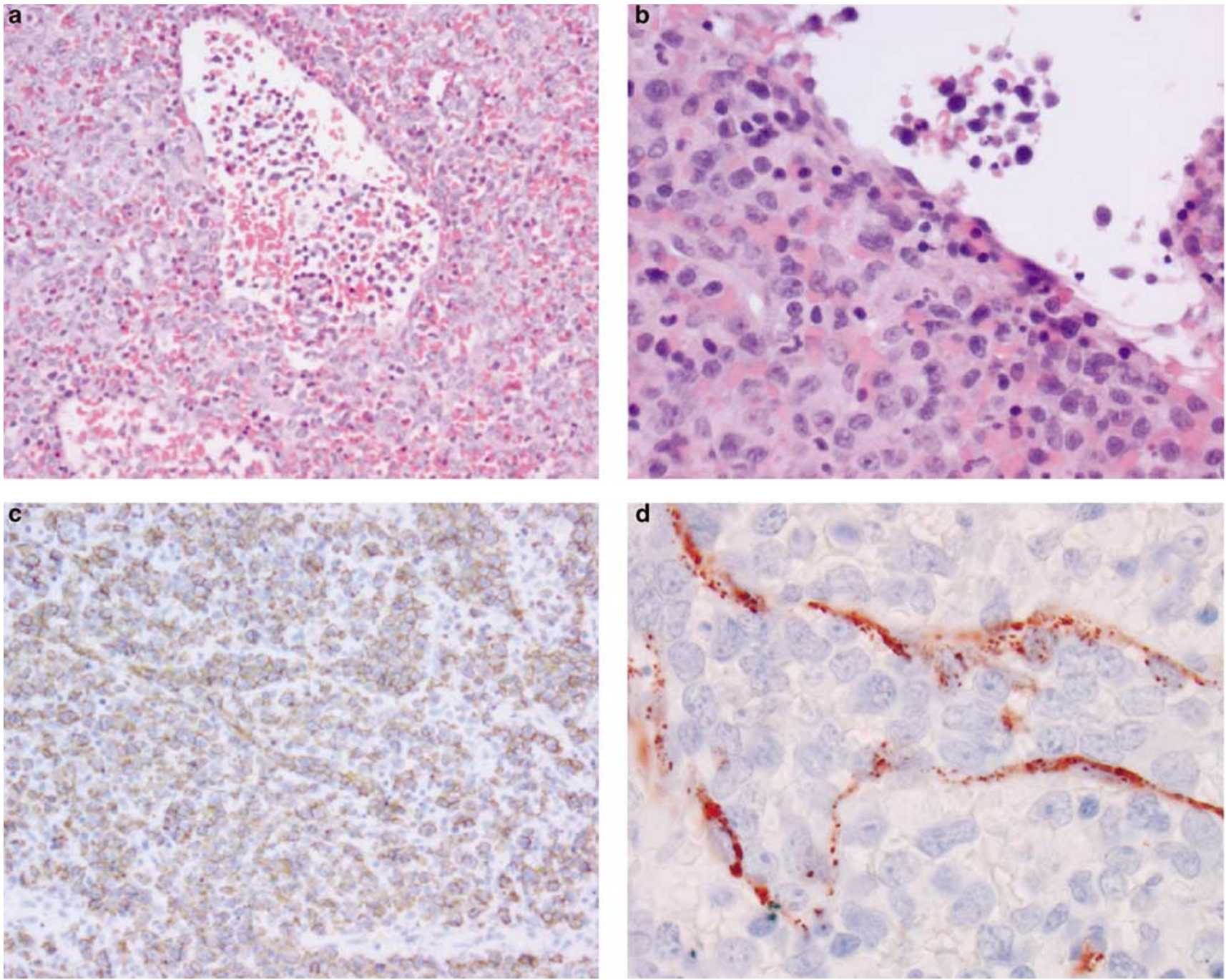

Figure 2 Spleen pathology: (a and b) Spleen H\&E-stained sections showing diffuse infiltration of the splenic red pulp by large B-cell lymphoma, which is most evident within the cords but is also present in the sinusoids (a, Patient $1, \times 200 ; \mathbf{b}$, Patient 2 , $\times 600$ oil). (c) CD20 immunoperoxidase staining highlights the presence of large B-cell lymphoma in the splenic cords and sinusoids (Patient $2, \times 200)$. (d) Factor VIII immunoperoxidase staining of the spleen illustrates distention of vascular structures by neoplastic cells (Patient $1, \times 400$ oil).

pronormoblasts similar in size and morphology to the LBCL cells (Figure 4f).

\section{Clinical Course}

Patient 1 had been treated with chemotherapy (ProMace/CYTABOM) prior to splenectomy and died 1 week following splenectomy (10 months after the onset of symptoms) secondary to respiratory failure. Patient 2 also received chemotherapy (CHOP) prior to splenectomy. At 1 month after splenectomy he developed recurrent emesis, nausea, and hypotension. An abdominal X-ray showed free air. The patient's condition continued to deteriorate and he died, presumably of bowel perforation and septic shock, 8 months after the initial onset of symptoms. An autopsy was not performed in either case.

\section{Discussion}

Splenic involvement by B-cell lineage lymphoproliferative disorders is not an infrequent occurrence. ${ }^{1}$ However, these are usually of low grade and they most often preferentially infiltrate the splenic white pulp. One notable exception is hairy cell leukemia, which is a red pulp-based low-grade B-cell lymphoproliferative disorders. ${ }^{9}$

In contrast to low-grade B-cell lymphoproliferative disorders, splenic involvement by diffuse LBCL is uncommon. When splenic involvement by LBCL occurs, it classically forms discrete tumor nodules within the parenchyma, although isolated 
descriptions of LBCL with diffuse red pulp involvement have appeared in the literature. ${ }^{2,10,11} \mathrm{~A}$ recent report of 33 splenic LBCL described three distinct patterns of lymphomatous infiltration: macronodular (or tumoral, 20 cases), micronodular (or miliary, nine cases), and diffuse (four cases). ${ }^{3}$ The diffuse pattern, which was the least common, described
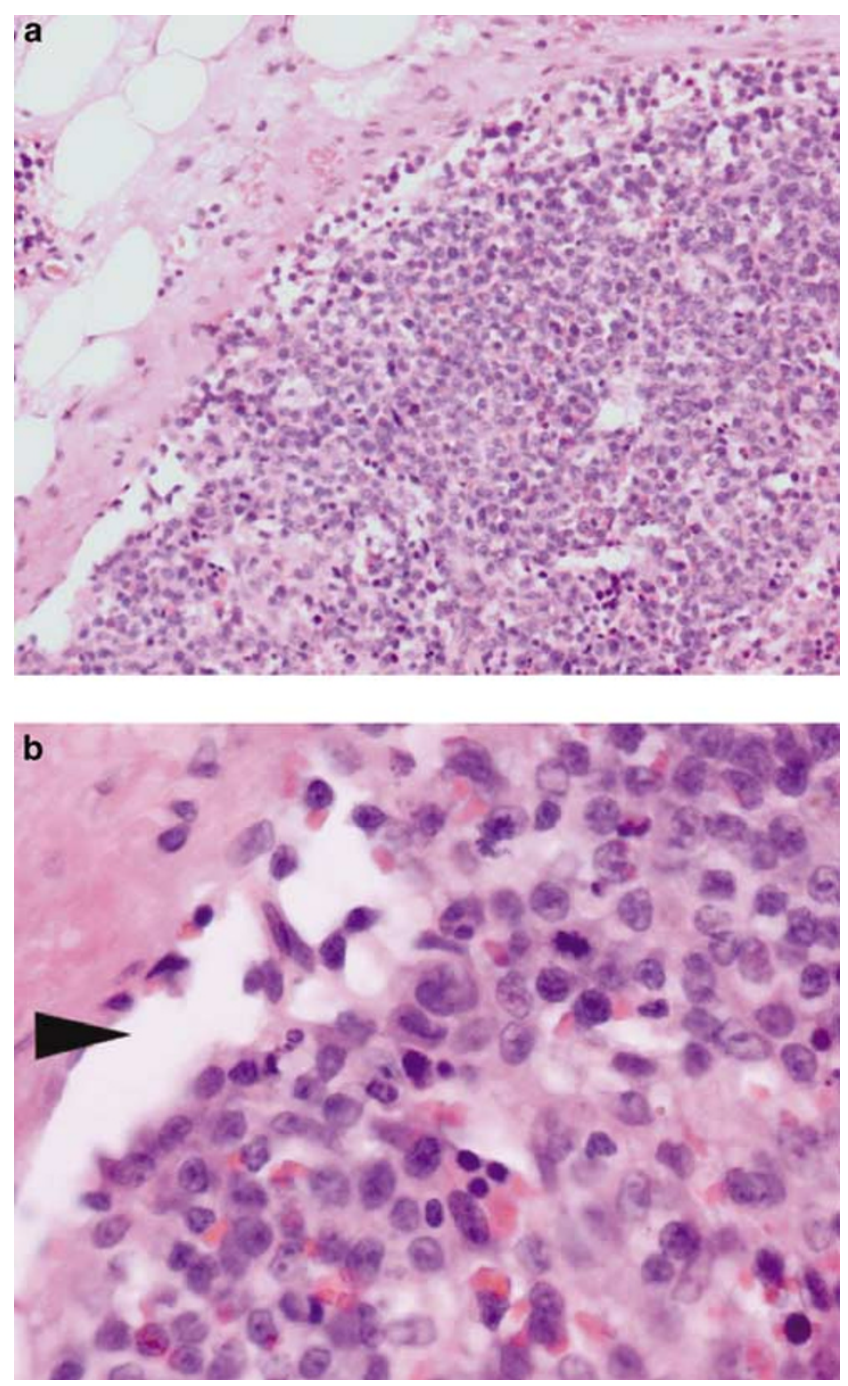

Figure 3 Lymph node pathology: (a) Low power view of a splenic hilar lymph node reveals architectural effacement by large B-cell lymphoma, which, at higher magnification (b) can be seen to spare the subcapsular sinus (arrow) (Patient $1, \mathrm{H} \& \mathrm{E}, \mathbf{a}, \times 200$; b, $\times 1000$, oil). cases of LBCL with involvement of the red pulp cords and sinusoids, similar to the pattern observed in this report. As in our patients, this pattern of splenic involvement by LBCL was associated with a high clinical stage and poor outcome. In two of these four reported cases of diffuse splenic LBCL involvement of splenic hilar lymph nodes was documented. Reportedly BM involvement was present in one of the four cases, with peripheral blood involvement in an additional two, although detailed descriptions of the peripheral blood and/or BM findings (including the presence or absence of cytopenias) are not provided. Given the difficulty in identifying marrow involvement by lymphoma by morphologic review in our patients with diffuse splenic LBCL, it is possible that marrow involvement was underreported in this antecedent study.

A number of other reports have also described predominant red pulp involvement in splenic LBCL. ${ }^{12-14}$ These include a purportedly distinct variant of intrasinusoidal splenic LBCL occurring primarily in Asians which is associated with histocytic proliferation, prominent hemophagocytosis, and aggressive behavior. ${ }^{15-17}$ The nature of these latter cases remains unclear and it has been speculated that some may represent a variant of intravascular LBCL.

Intravascular LBCL is an enigmatic disease in which the neoplastic cells are exclusively confined to the lumina of small vessels, possibly due to the lack of adhesion molecules required for tissue homing. ${ }^{18}$ This unusual distribution of lymphoma cells leads to protean clinical features secondary to small vessel occlusion including nephrotic syndrome, mental status changes, and shortness of breath. ${ }^{19,20}$ Hematologic abnormalities, including autoimmune hemolytic anemia, have also been associated with this diagnosis. ${ }^{4}$ Given the clinical features in our patients, including the presence of unexplained cytopenias and dyspnea, and the pattern of BM and splenic involvement a diagnosis of intravascular LBCL was considered. However, parenchymal involvement of splenic hilar nodes excluded this diagnosis when the most recent WHO criteria for this diagnosis were applied. ${ }^{4}$

An intrasinusoidal pattern of marrow infiltration has been described in intravascular LBCL as well as in a number of other lymphoproliferative disorders including splenic marginal zone B-cell lymphoma, hepatosplenic T-cell lymphoma, and T-cell large

Table 2 Bone marrow pathology

\begin{tabular}{llllcccc}
\hline & Cellularity & Eryth & Grans & Megs & Lymphoma H\&E & Lymphoma IHC & Lymphoma pattern \\
\hline Pt 1 & INC (75\%) & INC & NL & NL & $5 \%$ & $20 \%$ & Intrasinusoidal \& Interstitial \\
Pt 2 & INC (65\%) & INC & NL & INC & $10 \%$ & $20-30 \%$ & Intrasinusoidal \& Interstitial \\
\hline
\end{tabular}

Pt 1, patient 1; Pt 2, patient 2; INC, increased; NL, normal; Eryth, erythropoiesis; Grans, granulopoiesis; megs, megakaryopoiesis; lymphoma H\&E, percentage marrow involvement by lymphoma as estimated from morphology; lymphoma IHC, percentage marrow involvement by lymphoma as estimated from CD20 immunoperoxidase stain. 
granular lymphocytic leukemia. ${ }^{21-27}$ These latter disorders share as a unifying feature prominent splenic involvement. It is recognized that morphologic detection of intrasinusoidal marrow involve- ment by these disorders is exceedingly difficult. Indeed, in initial descriptions of intravascular LBCL, marrow involvement was described as an infrequent occurrence. ${ }^{19,28}$ However, subsequent
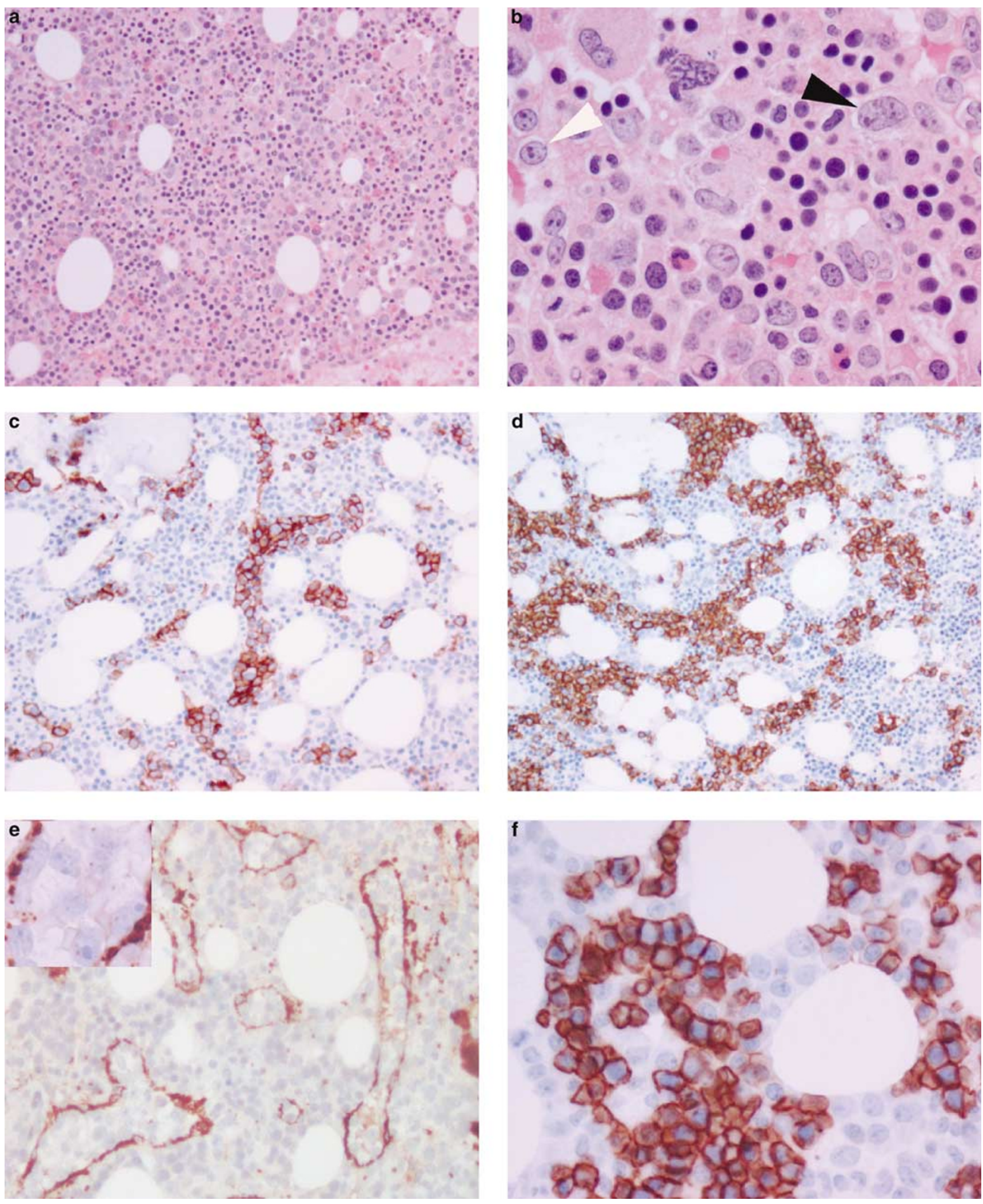
studies indicate that this disease involves the marrow with greater frequency than originally suggested..$^{20,25}$ In our patients with diffuse splenic involvement by LBCL, there was a predominant, but not exclusive intrasinusoidal pattern of marrow infiltration. Detection of marrow involvement by LBCL in our cases was greatly facilitated by antiCD20 immunoperoxidase staining as morphologic clues to the diagnosis were obscured both by the pattern of marrow involvement and the associated prominent erythroid hyperplasia. Both of these confounding elements are likely related to degree of splenic involvement by these malignancies; as spleen-based lymphoproliferative disorders tend to show intrasinusoidal marrow infiltration and the splenic enlargement caused could result in hypersplenism in turn leading to increased marrow hematopoiesis.

In summary, the cases of large B-cell lymphoma described in this report appear to be representative of an unusual, aggressive form of this disease with prominent splenic involvement that eludes prompt diagnosis due to the nonspecific nature of the clinical symptoms and the absence of mass lesions classically associated with splenic involvement by LBCL. Furthermore, these cases both presented with BM involvement that was obscured by a predominantly intrasinusoidal pattern of infiltration often associated with intravascular LBCL as well as the presence of an associated erythroid hyperplasia. Recognition of this morphologically subtle intrasinusoidal pattern of marrow infiltration in these unusual LBCLs is important as both patients presented with unexplained cytopenias and, as a result, in each BM biopsy was the first diagnostic tissue specimen obtained. Given the difficulties in clinical and histopathologic recognition of this disorder, as well as other lymphoproliferative disorders with significant splenic involvement, the use of ancillary immunoperoxidase stains should be considered in the evaluation of BM biopsies obtained from patients in which splenic lymphoma is a diagnostic consideration and yet morphologic features of lymphomatous involvement are not evident.

\section{References}

1 Arber DA, Rappaport H, Weiss LM. Non-Hodgkin's lymphoproliferative disorders involving the spleen. Mod Pathol 1997;10:18-32.
2 Harris NL, Aisenberg AC, Meyer JE, et al. Diffuse large cell (histiocytic) lymphoma of the spleen. Clinical and pathologic characteristics of ten cases. Cancer 1984; 54:2460-2467.

3 Mollejo M, Algara P, Mateo MS, et al. Large B-cell lymphoma presenting in the spleen: identification of different clinicopathologic conditions. Am J Surg Pathol 2003;27:895-902.

4 Gattner KCWR. Intravascular large B-cell lymphoma In: Jaffe ES, Stein H, Vardiman JW (eds). Classification of Tumours. Pathology and Genetics of Tumours of Haematopoietic and Lymphoid Tissues. IARC Press: Lyon, 2001, pp 177-178.

5 Kurtin PJ, Hobday KS, Ziesmer S, et al. Demonstration of distinct antigenic profiles of small B-cell lymphomas by paraffin section immunohistochemistry. Am J Clin Pathol 1999;112:319-329.

6 Hanson CA, Kurtin PJ, Katzmann JA, et al. Immunophenotypic analysis of peripheral blood and bone marrow in the staging of B-cell malignant lymphoma. Blood 1999;94:3889-3896.

7 Chang KL, Chen YY, Shibata D, et al. Description of an in situ hybridization methodology for detection of Epstein-Barr virus RNA in paraffin-embedded tissues, with a survey of normal and neoplastic tissues. Diagn Mol Pathol 1992;1:246-255.

8 Colomo L, Lopez-Guillermo A, Perales $\mathrm{M}$, et al. Clinical impact of the differentiation profile assessed by immunophenotyping in patients with diffuse large B-cell lymphoma. Blood 2003;101:78-84.

9 Hoyer JD, Li CY, Yam LT, et al. Immunohistochemical demonstration of acid phosphatase isoenzyme 5 (tartrate-resistant) in paraffin sections of hairy cell leukemia and other hematologic disorders. Am J Clin Pathol 1997;108:308-315.

10 Betman HF, Vardiman JW, Lau J. T-cell-rich B-cell lymphoma of the spleen. Am J Surg Pathol 1994; 18:323-324.

11 Faravelli A, Gambini S, Perego D, et al. Splenic lymphoma: unusual case with exclusive red pulp involvement. Pathologica 1995;87:692-695.

12 Palutke M, Eisenberg L, Narang S, et al. B lymphocytic lymphoma (large cell) of possible splenic marginal zone origin presenting with prominent splenomegaly and unusual cordal red pulp distribution. Cancer 1988;62:593-600.

13 Salgado C, Feliu E, Montserrat E, et al. B-type large-cell primary splenic lymphoma with massive involvement of the red pulp. Acta Haematol 1993;89:46-49.

14 Kobrich U, Falk S, Karhoff M, et al. Primary large cell lymphoma of the splenic sinuses: a variant of angiotropic B-cell lymphoma (neoplastic angioendotheliomatosis)? Hum Pathol 1992;23:1184-1187.

15 Murase T, Nakamura S, Kawauchi K, et al. An Asian variant of intravascular large B-cell lymphoma: clinical, pathological and cytogenetic approaches to

Figure 4 Bone marrow pathology: (a) Hypercellular bone marrow with erythroid hyperplasia, involvement by large B-cell lymphoma is not readily evident (Patient $2, \times 200$ ). (b) Higher magnification reveals subtle interstitial infiltration by atypical lymphoid cells with large vesicular nuclei (black arrow) which are morphologically similar to the early erythroid precursors (white arrow) (Patient $2, \times 600$, oil). (c and d) CD20 immunoperoxidase staining demonstrates a predominantly intrasinusoidal pattern of marrow infiltration by large B-cell lymphoma in both cases (c, Patient 2, $\times 400$ oil; d, Patient $1, \times 300$ ). (e) Factor VIII immunoperoxidase staining highlights distention of bone marrow sinusoids by cells which at higher magnification (inset) are seen to be neoplastic cells with large nuclei and prominent nucleoli (Patient $2, \times 200$, inset $\times 500$ oil). (f) Immunoperoxidase staining with antibodies to glycophorin $1 \mathrm{~A}$ confirms the increase in erythroid precursors and illustrates the similarity between the early erythroid precursors and the large lymphoma cells, which are glycophorin 1A negative (Patient $2, \times 600$, oil). 
diffuse large B-cell lymphoma associated with haemophagocytic syndrome. Br J Haematol 2000;111:826-834.

16 Murase T, Nakamura S, Tashiro K, et al. Malignant histiocytosis-like B-cell lymphoma, a distinct pathologic variant of intravascular lymphomatosis: a report of five cases and review of the literature. Br J Haematol 1997;99:656-664.

17 Dufau JP, Le Tourneau A, Molina T, et al. Intravascular large B-cell lymphoma with bone marrow involvement at presentation and haemophagocytic syndrome: two Western cases in favour of a specific variant. Histopathology 2000;37:509-512.

18 Ponzoni M, Arrigoni G, Gould VE, et al. Lack of CD 29 (Beta1 integrin) and CD 54 (ICAM-1) adhesion molecules in intravascular lymphomatosis. Hum Pathol 2000;31:220-226.

19 DiGiuseppe JA, Nelson WG, Seifter EJ, et al. Intravascular lymphomatosis: a clinicopathologic study of 10 cases and assessment of response to chemotherapy. J Clin Oncol 1994;12:2573-2579.

20 Calamia KT, Miller A, Shuster EA, et al. Intravascular lymphomatosis. A report of ten patients with central nervous system involvement and a review of the disease process. Adv Exp Med Biol 1999;455:249-265.

21 Farcet JP, Gaulard P, Marolleau JP, et al. Hepatosplenic T-cell lymphoma: sinusal/sinusoidal localization of malignant cells expressing the T-cell receptor gamma delta. Blood 1990;75:2213-2219.
22 Morice WG, Kurtin PJ, Tefferi A, et al. Distinct bone marrow findings in T-cell granular lymphocytic leukemia revealed by paraffin section immunoperoxidase stains for CD8, TIA-1, and granzyme B. Blood 2002; 99:268-274.

23 Vega F, Medeiros LJ, Bueso-Ramos C, et al. Hepatosplenic gamma/delta T-cell lymphoma in bone marrow. A sinusoidal neoplasm with blastic cytologic features. Am J Clin Pathol 2001;116:410-419.

24 Audouin J, Le Tourneau A, Molina T, et al. Patterns of bone marrow involvement in 58 patients presenting primary splenic marginal zone lymphoma with or without circulating villous lymphocytes. Br J Haematol 2003;122:404-412.

25 Estalilla OC, Koo CH, Brynes RK, et al. Intravascular large B-cell lymphoma. A report of five cases initially diagnosed by bone marrow biopsy. Am J Clin Pathol 1999;112:248-255.

26 Tucker TJ, Bardales RH, Miranda RN. Intravascular lymphomatosis with bone marrow involvement. Arch Pathol Lab Med 1999;123:952-956.

27 Costes V, Duchayne E, Taib J, et al. Intrasinusoidal bone marrow infiltration: a common growth pattern for different lymphoma subtypes. Br J Haematol 2002; 119:916-922.

28 Wick MR, Mills SE. Intravascular lymphomatosis: clinicopathologic features and differential diagnosis. Semin Diagn Pathol 1991;8:91-101. 